\title{
Performance of Video Conferencing using Protocol Independent Multicast Routing with Core failure
}

\author{
V. Chandrasekar \\ CSE Department, \\ Dhanalakshmi Srinivasan Engineering College, \\ Perambalur-621212 \\ Tamilnadu, India
}

\author{
K. Baskaran \\ CSE Department, \\ Government College of Technology, \\ Coimbatore \\ Tamilnadu, India
}

\begin{abstract}
Video conferencing is now widely used both in wired and wireless network. Quality of Service (QoS) depends upon the efficient utilization of the network bandwidth. Internet Protocol (IP) multicasting is simultaneous transmission of data to multiple destination. IP multicast uses one-to-many technique, wherein a single packet is sent to multiple destinations in a multicast group identified by a single IP destination group address. Core failure is a serious issue in multicast networks with the QOS diminishing even if alternative routes are available. This paper investigates the performance of streaming data which require stringent QOS using unicast and multicast communication with Protocol Independent Multicasting - Sparse Mode (PIM-SM) . An intermediate core is failed and the performance of the network measured. Results obtained show the degradation due to core failure affects the QOS for streaming data.
\end{abstract}

\section{Keywords}

Unicast, Multicast, Protocol Independent Multicast - Sparse Mode, Internet Group Management Protocol.

\section{INTRODUCTION}

Traditionally, unicast transmissions are used for network processes in which packets are sent from host to host. Thus, when a unicast transmission is used to send packet to multiple destinations, it ends up crossing the same links repetitively. Broadcast transmission is used when all the nodes in the network are recipients. Multicasting is simultaneous transmission of packets to multiple intended destinations, and is used in many multimedia applications [1]. The multicast group is generally identified by a single IP destination group address. Anycast is when data is to be transmitted to any one of the members selected to be part of a group[2].

During multicast sessions, minimal network resources must be utilized for transport of data. Tree construction is the most popular way of multicast routing. A multicast tree is created during a multicast session through which the data is transferred. The multicast tree is constructed using specific algorithms called multicast routing algorithm. Applications that are simultaneously synchronized require a good amount of reserved resources to maintain Quality of Service (QoS). Typical QOS parameters include end-to-end delay, delay variation, loss, cost and throughput to name a few [2]. In a multicast tree, resources are reserved along path to each destination, thus, during construction of tree if required QoS in a single link is insufficient then the algorithms fails to construct the tree. For efficient multicast communication, it is required to build a multicast tree which has the best chance to satisfy the resource requirements [3]. The parameters that measure the Quality of Services are Delay, Jitter, Bandwidth and Reliability. Two basic types of protocols are available to satisfy the varieties of application needs are

\section{- Resource Reservation (RSVP) (Integrated Services) \\ - $\quad$ Prioritization (DSCP) (Differentiated Services)}

The RSVP protocol is used by a host to request specific qualities of service from the network for particular application data streams or flows and to deliver QoS requests to all nodes along the paths of the flows and to establish and maintain state to provide the requested service.

Differentiated Services provide a per-flow state and signaling in every router and hence offers a wide range of services[14].

For multicast routing algorithms, tree construction are based either on Source Based Algorithms(SBA) or Core Based Algorithms(CBA)

Source Based Algorithms (SBA) algorithms construct a tree from the source branching out to all the destinations in the multicast group, messages are transmitted through the tree. Each node in the network maintains a global state. Protocol Independent Mode Dense Mode (PIM-DM) and Multicast Open Shortest Past First (MOSPF) are based on SBA.

Core based Algorithms (CBA) is used in many-to-many multicasting scenario. In CBA, a core node is selected which forms the root of the multicast tree. The tree branches out from the core node to all the members in the group. The QoS of the tree depends on the core node, so core nodes are required to be chosen with care. Core Based Tree (CBT) and Protocol Independent Multicast Sparse Mode (PIM-SM) are based on CBA.

A Communication network failure can have an adverse effect on today's society. In the future, as more applications employ multicast routing, a strong need will emerge for algorithms that can be employed by survivable multicast routing protocols. Regarding core-based multicasting, the main problem is that it has a single point of failure at the core. If the core fails, the 
whole multicast session would be disrupted. To provide reliable multicast services, the multicast routing protocols need to be equipped with mechanisms for handling core failures as well as node/link failures. The following issues are associated with core failure recovery [15].

- Core selection and tree construction: A new core must be selected from a list of candidate cores with a multicast tree that minimizes service disruption and tree cost.

- Local recovery: Once a core failure or link/node failure has occurred, recovery may take place on a local scale whereby nodes contact other nearby nodes and perform local rerouting.

- Global recovery: In the event of a severe failure, it may be necessary to globally recover the multicast group. For these instances, the new core must be evaluated and the members of the multicast tree must be migrated to the new tree.

In this paper it is proposed to evaluate the performance of multicasting in a sparse network using Protocol Independent Multicasting Sparse Mode (PIM-SM) under different link scenarios with core failure and as well as node/link failures.

\section{LITERATURE REVIEW}

A multicast tree is generally implemented using a Steiner tree $[4,5]$. Constructing a multicast tree with minimal cost is a NPhard problem. Kou et al., proposed the Kou, Markowsky and Berman (KMB) algorithm [6]

where each node represents either a source or destination with the edge cost being lowest among all paths connecting the two nodes. The network

is built as a complete graph. Prims algorithm [7] is used to find the Minimum Spanning Tree (MST). The major drawback of KMB algorithm is that the QoS issues are not addressed.

Kompella et al., proposed Kompella, Pasquale and Polyzos (KPP) algorithm [8] to address the QOS parameters. To achieve better QOS, the delay constraint requirement was maintained for a specific threshold and parameters measured using link cost and link delay. The tree construction is based on a near Minimum Cost Tree such that the delay between every two destination nodes is less than the specified threshold, the link delays are assumed to be integers and delay constraint is bounded, so that the complete graph can be constructed in polynomial time.

Kumar et.al [12] proposed two routing trees: a shortest path tree and a Steiner tree. It identifies a given number of $\mathrm{k}$ destinations, the difference between the delay observed in the Steiner tree and the delay in the shortest path tree is largest and such destinations are replaced from the Steiner tree to the shortest path tree.

The constrained Bellman Ford Algorithm [12] is used to connect one group member at a time to the source. It is based on Constrained Adaptive Ordering Heuristic that states that after each run the unconnected member with the delay constrained minimum-cost path to the source is chosen and added to the existing sub-tree.

Multiple Adaptive Multiple Constraints Routing Algorithm [12] guarantees QoS to the multicast members in an efficient, but not always optimal manner and can be considered as a heuristic to solve multiple parameters Steiner tree. It constructs the set of shortest paths from the source to all the multicast members are first evaluated and the entire tree is optimized such that the length function is reduced, without violating the constraints.

DVMA builds a DVBMT[13] spanning tree and is an NPcomplete problem. The algorithm starts with a spanning tree satisfying the delay constraint, which may not include some members, the algorithm searches through the candidate paths satisfying the delay and the delay variation constraint from a non-tree member node to any one of the tree nodes. On finding such a path, it adds the members to the existing tree. DVMA satisfies the variation constraint or has the smallest value of variation among the trees considered by the algorithm.

Bounded Shortest Multicast (BSM) algorithm [9] is based on the Shortest Path Tree (SPT). This method find the shortest end to end delay path to solve the multicast problem. An SPT is built and refined recursively by replacing the $f$ branching nodes with lower cost branching nodes. The branching nodes are also called as super edges.

\section{PROPOSED METHODOLOGY}

Video conferencing applications to guarantee quality of service have to deliver data with minimum jitter and end to end delay. The challenges increase many fold when the last mile could be either wired or wireless. Multicast communication improves the QOS compared to unicast due to the reduced data duplication and hence better utilization of bandwidth. In this paper it is proposed to study the effect of core failure in the performance of multicast systems. Figure 1 shows the architecture of the proposed system. The experimental setup consists of about 30 receivers located at various geographical locations and interconnected by three core routers connected in star topology. Two scenarios are considered in this work with the core failure scenario consisting of link breakage among the cores as shown in figure 2.

Simulations were carried out for 300 seconds with video conferencing happening simultaneously among various systems in the network and the simulation parameters measured.

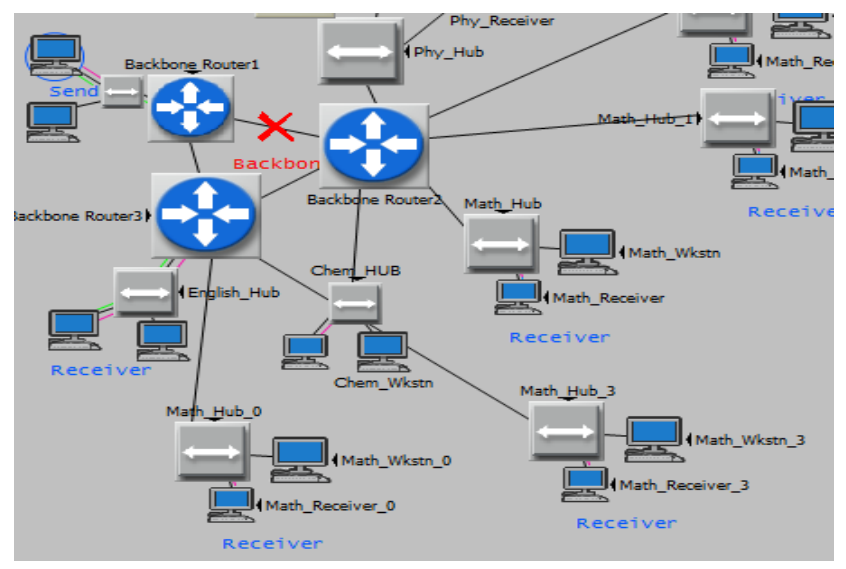

Figure 1 : Experimental set up with core failure indicated using red mark. 


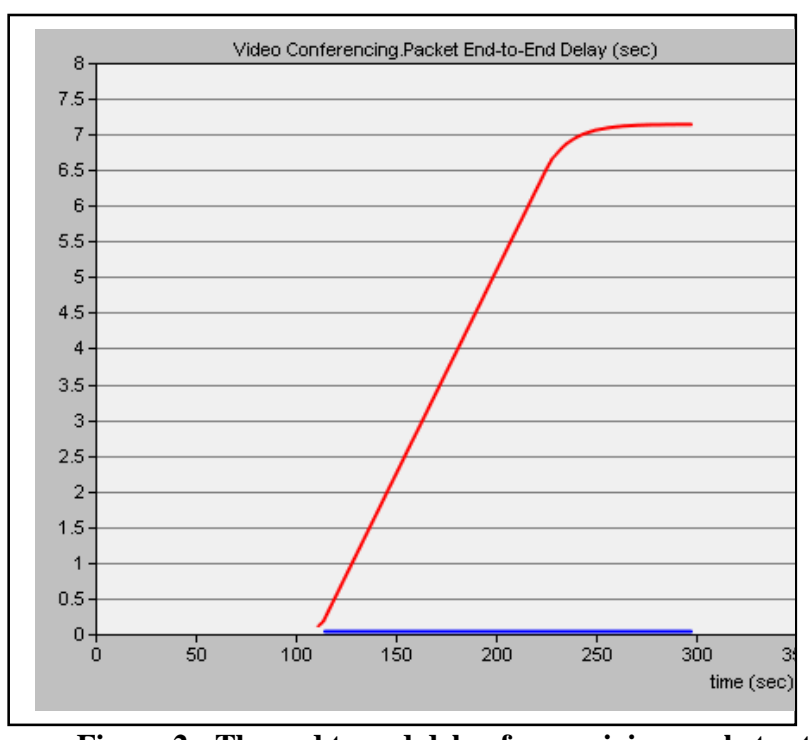

Figure 2 : The end to end delay for receiving packets at core 3 subnet.

\section{RESULT AND DISCUSSION}

Two specific systems were considered for measuring the QOS parameters. One of the video conferencing node is located in the subnet of core 2 and another in the subnet of core 3 . These two systems were considered due to the additional traffic carried out by core 2 in the event of link

failure between core 1 and core 2 . The end to end delay of the network due to core failure for the

system located in sub network of core 3 is shown in figure 2 . From figure 2 it is seen that the end to end delay increases linearly when the core fails ( indicated by red ). This can definitely affect the quality of reception and transmission for nodes located in subnet of core 2 though the link between core 1 and core 3 is good. Figure 3 shows the end to end delay for systems in core 2 subnet. The end to end delay increases by 6 milli second which will not affect the quality of video conferencing reception. This delay occurs when the additional hop required to traverse from core 1 to core 3 . Table 1 shows the actual delay.

\section{Table 1: End To End Delay}

\begin{tabular}{|l|l|l|}
\hline $\begin{array}{l}\text { Simulation time in } \\
\text { second }\end{array}$ & $\begin{array}{l}\text { end to end delay in core } \\
\text { 3 subnet in second }\end{array}$ & $\begin{array}{l}\text { end to end delay in core 2 } \\
\text { subnet in second }\end{array}$ \\
\hline 0 & 0 & 0 \\
\hline 100 & 0.035082231 & 0.197519311 \\
\hline 150 & 0.035082094 & 2.245076542 \\
\hline 200 & 0.035082094 & 5.146027083 \\
\hline 250 & 0.035082094 & 7.055541764 \\
\hline 300 & 0.035082094 & 7.138478724 \\
\hline
\end{tabular}

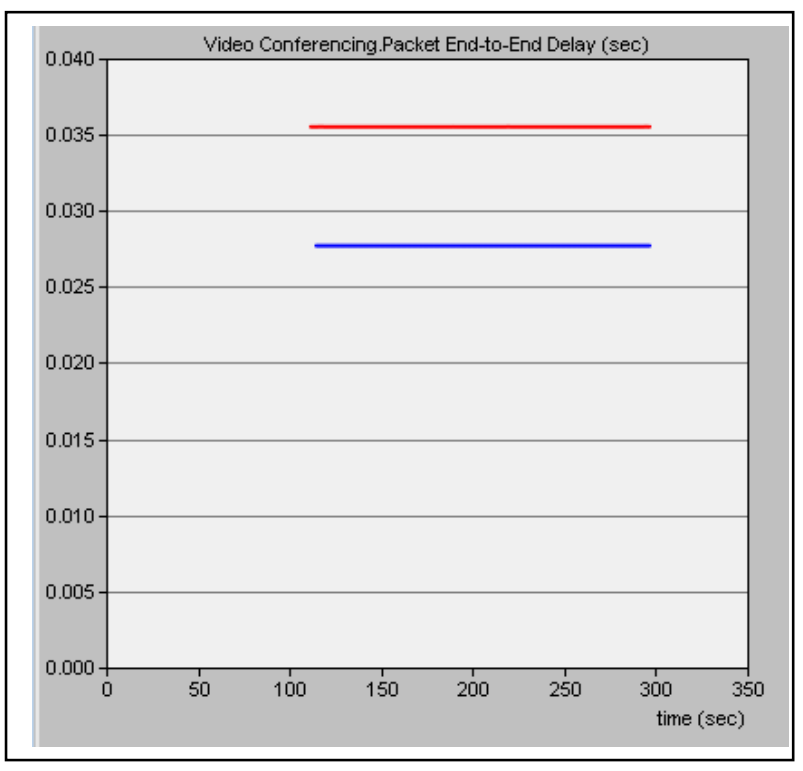

Figure 3 : End to end delay at system located in sub network of core 2

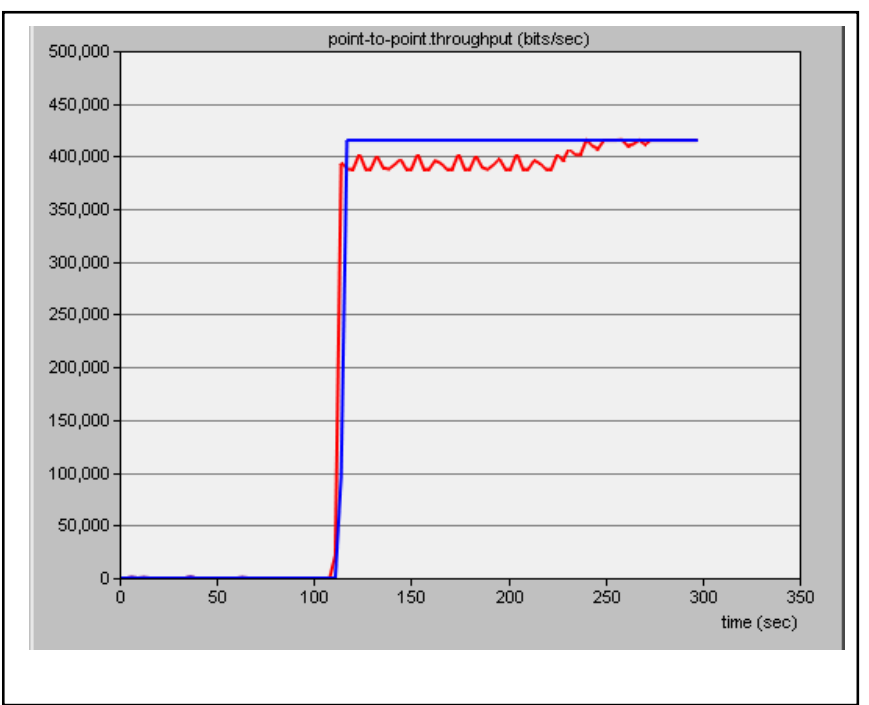

Figure 4 : The throughput of the network between core 1 and core 3 . 
The throughput of the system during normal operation and core failure is shown in figure 4.

From figure IV it is seen that the throughput during the core failure consists of jitter which can affect the quality of received transmission.

\section{CONCLUSION}

In this paper it was proposed to study the effect of core failure during video conferencing under multicasting in a sparse network using Protocol Independent Multicasting Sparse Mode (PIM-SM). The end to end delay increases by an average of 4.7 seconds in the sub network of core 3 due to link failure between core 1 and core 2 . However the end to end delay increases by $6 \mathrm{~ms}$ in the systems at core network 2. The output is significant as the quality of the video conference decreases in the area where the link failure has not occurred.

\section{REFERENCES}

[1] A. Ballardie "Core Based Trees (CBT) Multicast Routing Architecture", RFC 2201, Internet Engineering Task Force, Sep 1997.

[2] Chen, S. \& Nahrsted, K., "An Overview of Quality of Service Routing for Next Generation High-speed Networks: Problems and Solutions", IEEE Network, November/December 1998 .

[3] Wang, B. \& Hou, J.C., "Multicast routing and its QoS extension: problems, algorithms, and protocols", IEEE Network Volume: 14 1, Jan.-Feb. 2000 , Page(s): 22 36.

[4] Makoto Imase and Bernard M. Waxman, "Dynamic Steiner Tree Problem," SIAM J. Discrete Math., 4(3), 1991, pp. 369-384.

[5] L. Zosin and S. Khuller, "On Directed Steiner Trees," SODA'02, pp.59-63.

[6] L. Kou, G. Markowsky and L. Berman, "A Fast Algorithm for Steiner Trees," Acta Informatica, vol. 15, 1981, pp. 141-145.

[7] T.H. Cormen, C.E. Leiserson and R.L. Rivest, Introduction to Algorithms,MIT Press, 1990.

[8] V.P. Kompella, J.C. Pasquale and G.C. Polyzos, "Multicast Routing for Multimedia Communication," IEEE/ACM Trans. Networking, June 1993,pp. 286292.
[9] Q. Zhu, M. Parsa and J. Garcia-Luna-Aceves, “A SourceBased Algorithm for Delay-Constrained MinimumCost Multicasting," IEEE INFOCOM,1995, pp. 377385 .

[10] RFC 2362 - Protocol Independent Multicast-Sparse Mode (PIM-SM)

[11] Wireless LAN Medium Access Control (MAC) and Physical Layer (PHY) Specification, IEEE Std. 802.11, 1997.

[12] Pragyansmita Paul and S V Raghavan "Survey of Multicast routing Algorithms and Protocols"

[13] G.N. Rouskas, I. Baldine, Multicast routing with end-toend delay and delay variation constraints, IEEE J.Select. Areas Commun. 15(3)(1997)346-356.

[14] Mansoor Mohsin, Winnie Wong, and Yogesh Bhatt "Support for Real- Time Traffic in the Internet, and Qos Issues.

[15] Aaron Striegel and G. Manimaran, lowa State University "A Survery of Qos Mutlicasting Issues" IEEE Communications Magazine, June 2002, pp82-87.

\section{AUTHOR PROFILE}

Dr. K. Baskaran received his Bachelor of Engineering degree in Electrical and Electronics Engineering from the Annamalai University, India in 1989 , Master of Engineering degree in Computer Science Engineering from Bharathiar University, India in 2002.and $\mathrm{Ph}$. D degree from Anna University-Chennai, India in 2006. He is a member of IEEE and member of ISTE..He is now Assistant Professor in Computer Science and Engineering, Government college of Technology, Coimbatore, India . His area of interest includes Adhoc networks, network security etc.

Mr. V.Chandrasekar received his Bachelor of Engineering degree in Computer Science \& Engineering from Bharathidasan University, Trichy,India in 1998, Master of Technology in Computer Science \& Engineering from SASTRA University, Thanjavur, India in 2004. He is pursuing Ph.D in Anna University, Chennai, India. He is a member of ISTE and ACM and he is now working as Professor in Computer Science \& Engineering, Dhanalakshmi Srinivasan Engineering College, Perambular, India. His area of interest includes Computer Networks, Routing Algorithms and Object Oriented Technology. 